\title{
Visual Outcome of Pars Plana Vitrectomy for Treatment of Premacular Hemorrhage Ali A Ghali ${ }^{1}$, Ashraf M Gad Elkareem ${ }^{2}$, Mohamed Al-Taher AA ${ }^{3}$ \\ Ophthalmology department, Faculty of medicine, Al-Azhar University, Damietta ${ }^{1}$, Asyut $^{2}$, and Cairo3Branches, Egypt. \\ Corresponding author: Ashraf Mohammed Gad Elkareem, Email: silicon2030@gmail.com, Tel: +2 01060293796
}

\begin{abstract}
Background: premacular hemorrhage can lead to painless loss of central vision. Several modalities of treatment have been described in previous reports.

Purpose: to evaluate the visual outcome after pars plan vitrectomy (PPV) for treatment of premacular hemorrhage of different etiology.

Patients and methods: A series of retrospective study 17 eyes of 17 patients presented with premacular hemorrhage due to different pathologies. Complete ophthalmic evaluation was done including visual acuity (VA), intraocular pressure (IOP), anterior segment examination, slit lamp biomicroscopy, fundus photography \pm fluorescein angiography (FA), and ocular ultrasound \pm biometry. PPV was performed using 23gauge system with proper tamponade \pm Phaco with PCIOL.No intraoperative lens touch or iatrogenic retinal tear has been recorded.

Results: BCVA was improved from CF $50 \mathrm{~cm}$ in 2 cases, hand movement (HM) in 15 cases to 0.1 (Log MAR) in 2 eyes (11.76\%), 0.3 in one eye (5.88\%), 0.4 in 5 eyes (29.41\%), 0.6 in 3 eyes (17.64\%), 1.00 in 3 eyes $(17.64 \%),(3 / 60)$ in 2 eyes (11.67\%), CF (1/60) in one eye (5.88\%). No serious complications weredetected.

Conclusion: PPV is a beneficial surgical treatment for premacular hemorrhage of different pathologies ensuring rapid visual recovery. The visual outcome is an encouraging and the complications are comparable to other studies.
\end{abstract}

Keywords: premacular hemorrhage, Nd: YAG laser, vitrectomy.

\section{INTRODUCTION}

Different pathologies can lead to premacular hemorrhage, which is either subhyaloid or sub-internal limiting membrane (ILM) hemorrhage. Retinal artery macroaneurysm, ocular trauma, proliferative diabetic retinopathy, acute posterior vitreous detachment, Valsalva retinopathy, retinal artery or vein occlusion, Terson syndrome, and shaken baby syndrome are probable causes of premacular hemorrhage which can occur spontaneously ${ }^{(1-4)}$. The biomicroscopic differentiation between the two subtypes is difficult, however the subhyaloid entity is characterized with a sharply delineated, hemispherical-shaped hemorrhage while the sub-ILM hemorrhage is characterized by its glistening striated surface ${ }^{(5,6)}$. OCT scans above the precipitated blood level can reveal the sub-ILM hemorrhage by the presence of, two membranes, a highly reflective one corresponding to the ILM and an anterior low reflective one corresponding to the posterior vitreous face ${ }^{(7)}$.

Either subhyaloid or sub-ILM premacular hemorrhage can lead to painless loss of central vision ${ }^{(8)}$. Several modalities of treatment weredescribed in previous studies. Waiting for spontaneous resolution is an option but it may take several months to resolve the hemorrhage during which time permanent macular damage can occur due to toxicity of the blood to the photoreceptors, also by the formation of epimacular membranes with macular tractional detachment ${ }^{(9,10)}$.
Opening the posterior vitreous face or the ILM by the $\mathrm{Nd}$ :YAG laser is another option for the treatment of premacular hemorrhage, allowing the drainage of the subhyaloid or sub-ILM hemorrhage into the vitreous cavity with a rapid gain of vision ${ }^{(1,2,4,8,11)}$.

Few complications of this maneuver as retinal break, macular hole, or retinal detachment can occur especially if the size of the blood in front of the macula is small ${ }^{(4)}$.

Pars plana vitrectomy can be done as early as possible for rapid visual recovery and to ensure complete surgical separation of the posterior vitreous face and clearance of the whole hemorrhage.

\section{AIM OF THE STUDY}

It is to evaluate the outcome of pars plana vitrectomy for clearance of premacular hemorrhage.

\section{PATIENTS AND METHODS}

A retrospective study of 17 eyes of 17 patients presented with premacular hemorrhage due to different causes was carried out. the demographic data of patients are shown in table (1). The study was done after the approval of the Research and Ethical Committee, School of Medical Sciences, Al-Azhar University, Egypt.

Complete ophthalmic evaluation was done including visual acuity (VA) measurement and was converted to Log MAR values, intraocular pressure (IOP) measured with the applanation tonometer, anterior segment examination, slit lamp bio-microscopy using 90 
D lens, fundus photography \pm fluoresceinangiography (FA), and ocular ultrasound and biometry in phakic eyes. OCT macula was done in selected cases. Written formal consent was obtained from all patients.

A standard 23gauge vitrectomy was done for all patients. Phaco-vitrectomy with PC IOL implantation was done in five out of nine phakic patients. The procedure was done under general anesthesia in 11 eyes and under peribulbar anesthesia in 6 eyes.After core vitrectomy triamcinolone acetonide (TAAc) was used in all cases to facilitate visualization of the vitreous and as anti-edematous particularly in diabetic cases. Induction of PVD was done using the suction mode of the vitrectomy cutter, the peripheral vitreous was shaved as much as possible, after complete separation of the posterior vitreous face, the premacular hemorrhage was sub-ILM in five cases in which the ILM was peeled first then the hemorrhage was aspirated. ILM peeling was done in 9 of 17 cases using 23gauge Eckhardt endgriping forceps and $0.1 \mathrm{ml}$ of a $0.06 \%$ brilliant blue stain.
In 3 cases hemorrhage was removed after peeling of the epiretinal membrane (ERM). Endo laser was applied in the form of pan retinal photocoagulation (PRP)using (double frequency) laser type in 3 cases having PDR. Tamponade was selected according to the retinal status at the end of surgery. Silicon oil was used in 2 cases and remained 6 months due to associated (PDR) and considerable risk of recurrent vitreous hemorrhage. $20 \%$ of SF6 was used ( $0.5 \mathrm{ML})$ in 8 eyes and air was used in 7 cases.No lens touch or iatrogenic retinal tear occurred during surgery as shown in table (3).

Subconjunctival injection of garamycin and dexamethasone was done in all cases. Postoperative systemic and topical antibiotic and anti-inflammatory have been prescribed for all cases for one week. Postoperative evaluation included BCVA, anterior segment assessment, IOP, slit lamp biomicroscopy in the first day postoperatively. After the first day the follow up scheduled to be weekly in the first month then every 6 weeks for 6 months postoperatively.

Tab (1): Demographic data of patients.

\begin{tabular}{|l|l|l|l|}
\hline No of eyes & \multicolumn{1}{|c|}{17} & & \\
\hline No of patients & \multicolumn{1}{|c|}{ Female } & \\
\hline Gender & Male & Fer & \\
& $9(52.94 \%)$ & $8(47.06 \%)$ & \\
\hline Age & Min & Max & Average \\
& 9.5 years & 62 years & 43.5 years \\
\hline Eye & Right & Left & \\
& $10(58.82 \%)$ & $7(41.18 \%)$ & Pseudophakic \\
\hline Lens status & Phakic & Aphakic & $6(35.29 \%)$ \\
& $9(52.94 \%)$ & $2(11.76 \%)$ & Valsalva \\
\hline Etiology & PDR & Trauma & $2(11.76 \%)$ \\
\hline & $7(41.18 \%)$ & $3(17.64 \%)$ & Unknown \\
& HTN & Vascular disorder & $1(5.88 \%)$ \\
\hline IVB (Avastin) & $2(11.76 \%)$ & $2(11.76 \%)$ & Postoperative \\
& $4(23.53 \%)$ & Intraoperative & $3(17.64 \%)$ \\
\hline Tamponade & Air & $3(17.64 \%)$ & Silicon oil \\
& $7(41.18 \%)$ & SF6 & $2(11.76 \%)$ \\
\hline Anesthesia & General & $8(47.06 \%)$ & \\
& $11(64.71 \%)$ & Peri-bulbar & \\
\hline Sclerotomies & Sutured & $6(35.29 \%)$ & Mixed \\
Sites & $6(35.29 \%)$ & Sutureless & $7(41.18 \%)$ \\
\hline Procedure & PPV & $4(23.53 \%)$ & \\
& $12(70.59 \%)$ & Phaco and PPV & \\
\hline
\end{tabular}

HTN $=$ hypertension. $\quad$ Mixed $=$ suturing only one or two sclerotomies Valsalva = Valsalva retinopathy. $\quad$ SF6 = sulfur hexafluoride gas. 


\section{Statistical analysis}

Categorical variables were described by number and percent $(\mathrm{N}, \%)$ and continuous variables were described by mean and standard deviation (Mean, SD). All analyses were performed with the SPSS 20.0 software (Inc., Chicago, IL, USA).

\section{RESULTS}

Preoperative BCVA was CF $50 \mathrm{~cm}$ in 2 cases, hand movement (HM) in 15 cases due to dense premacular hemorrhageand it improved to an average of $0.4 \pm 0.22 \log$ MAR in the first month post-vitrectomy. It was 0.1 (Log MAR) in 2 eyes $(11.76 \%), 0.3$ in one eye (5.88\%), 0.4 in 5 eyes (29.41\%), 0.6 in 3 eyes (17.64\%), 1.00 in 3 eyes (17.64\%), 3/60 in 2 eyes (11.67\%), and it was $1 / 60$ in one eye $(5.88 \%)$. The patients with reduced postoperative visual acuity(1/60-3/60) developed ischemic maculopathy and neovascular glaucoma. Table (2) shows the postoperative BCVA. The premacular hemorrhage was completely removed during vitrectomy after induction of PVD (Figures 1and 2).

Recurrent vitreous hemorrhage was observed in 2 eyes (11.67\%) after vitrectomy and has completely resolved by medical treatment in the form of aminocaproic acid and alpha chymotrypsin injection within 2-3 weeks. Complicated cataract to vitrectomy was reported in 4 eyes from 9 phakic (44.44\%) through the follow up time and 2 of them $(22.22 \%)$ had phacoemulsification and posterior chamber foldable intraocular lens (PC IOL) during the follow up period.

The intraocular pressure (IOP) was elevated (25$30 \mathrm{~mm} \mathrm{Hg}$ ) in 6 eyes $(35.29 \%)$ and controlled medically by topical combination of dorzolamide and timolol by the end of follow up period except one eye (5.88\%) remained on anti-glaucoma therapy(Table 4). Macular bucker was observed in 2 eyes $(11.76 \%)$ but no intervention was done.

Laser was performed in $3(17.64 \%)$ diabetic eyes in the form of (PRP) and macular grid during the follow up period due to progression of diabetic retinopathy. Repeated anti VEGF(Bevacizumab) injection was done in 2 eyes (11.67\%) during follow up time for treatment of diabetic macular edema and diabetic retinopathy. No early postoperative infection or bleeding were detected.

Tab (2): visual outcome after one month.

\begin{tabular}{|l|l|l|l|l|l|}
\hline NO of patients & $\%$ & BCVA & Log MAR & Procedure & Tamponade \\
\hline 2 & 11.76 & 0.80 & 0.10 & $\begin{array}{l}\text { PPV } \\
\text { PPV }\end{array}$ & $\begin{array}{l}\text { Air }(1 / 2) \\
\text { SF6 (1/2) }\end{array}$ \\
\hline 1 & 5.88 & 0.50 & 0.30 & Phaco & SF6 \\
\hline 5 & 29.41 & 0.40 & 0.40 & $\begin{array}{l}\text { Phaco+PPV } \\
\text { PPV }\end{array}$ & $\begin{array}{l}\text { AIR }(2 / 5) \\
\text { SF6 (3/5) }\end{array}$ \\
\hline 3 & 17.64 & 0.2 & 0.60 & PPV & $\begin{array}{l}\text { Air }(2 / 3) \\
\text { SF6 (1/3) }\end{array}$ \\
\hline 3 & 17.64 & 0.1 & 1.00 & Phaco + PPV & $\begin{array}{l}\text { SF6 (2/3) } \\
\text { Air }(1 / 2)\end{array}$ \\
\hline 2 & 11.67 & $0.05(3 / 60)$ & 1.3 & PPV & $\begin{array}{l}\text { Oil }(1 / 2) \\
\text { SF6 (1/2) }\end{array}$ \\
\hline 1 & 5.88 & CF (1/60) & 2.0 & Phaco + PPV & Oil \\
\hline
\end{tabular}

Tab (3): Intraoperative complications

\begin{tabular}{|l|l|l|}
\hline Intraoperative complications & No & \% \\
\hline bleeding & 2 & $11.67 \%$ \\
\hline Iatrogenic tear & 0 & $0 \%$ \\
\hline Phaco related complications & 0 & $0 \%$ \\
\hline Lens touch & 0 & $0 \%$ \\
\hline
\end{tabular}


Visual Outcome of Pars Plana Vitrectomy...

Tab (4): Postoperative complications

\begin{tabular}{|l|l|l|}
\hline Postoperative complications & No & Percentage (\%) \\
\hline Corneal edema & 1 & $5.88 \%$ \\
\hline Iritis & 1 & $5.88 \%$ \\
\hline Complicated cataract PPV & $4 / 9$ & $44.44 \%$ \\
\hline Elevated IOP & 6 & $35.29 \%$ \\
\hline Low IOP $(9 \mathrm{~mm} \mathrm{Hg})$ & 1 & $5.88 \%$ \\
\hline Recurrent vitreous hemorrhage & 2 & $11.76 \% \%$ \\
\hline
\end{tabular}

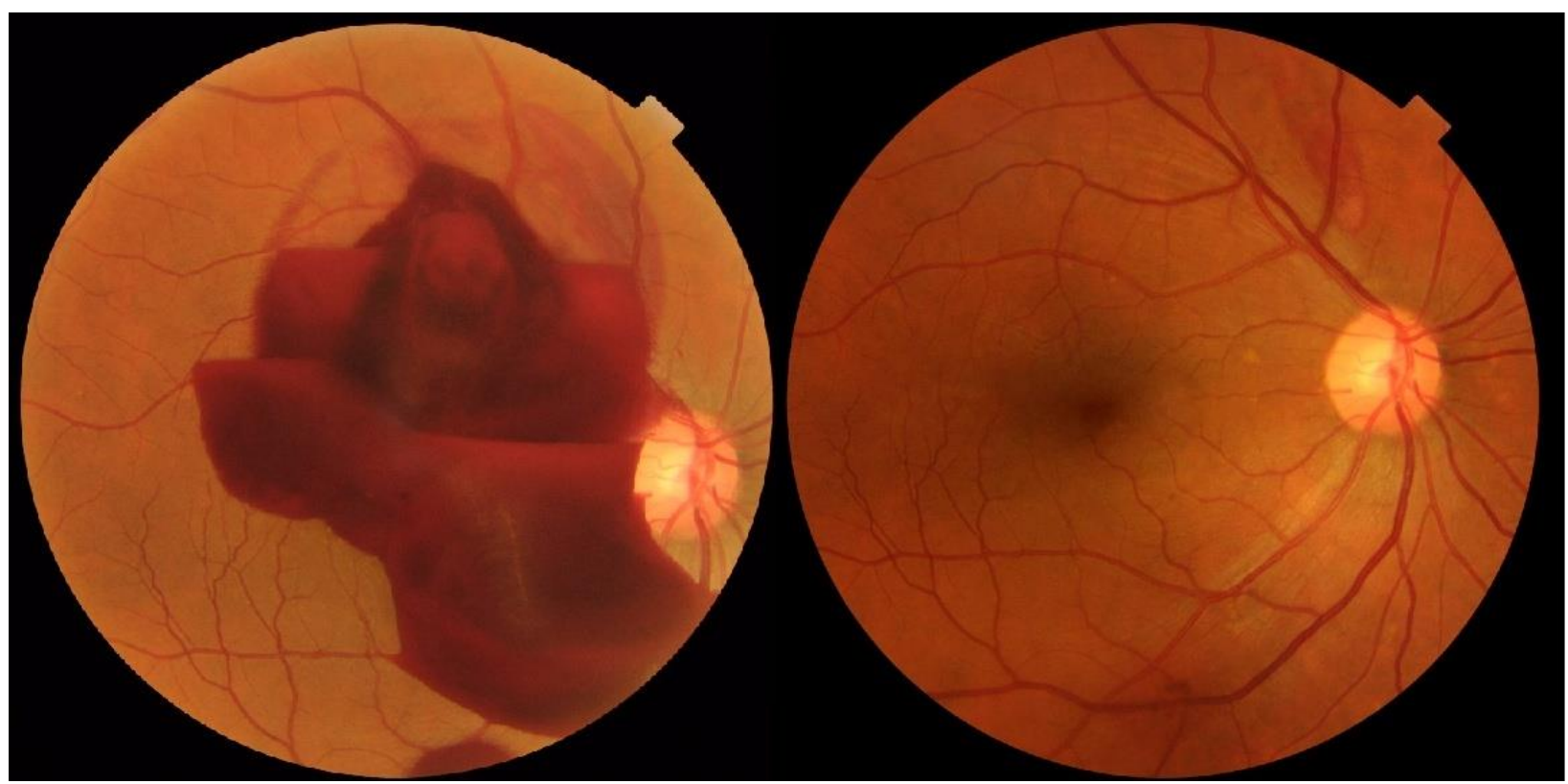

Figure 1: Fundus picture of the right eye of 45 years old male with premacular hemorrhage due to Valsalva maneuver (right image) which is completely resolved after vitrectomy (left image).

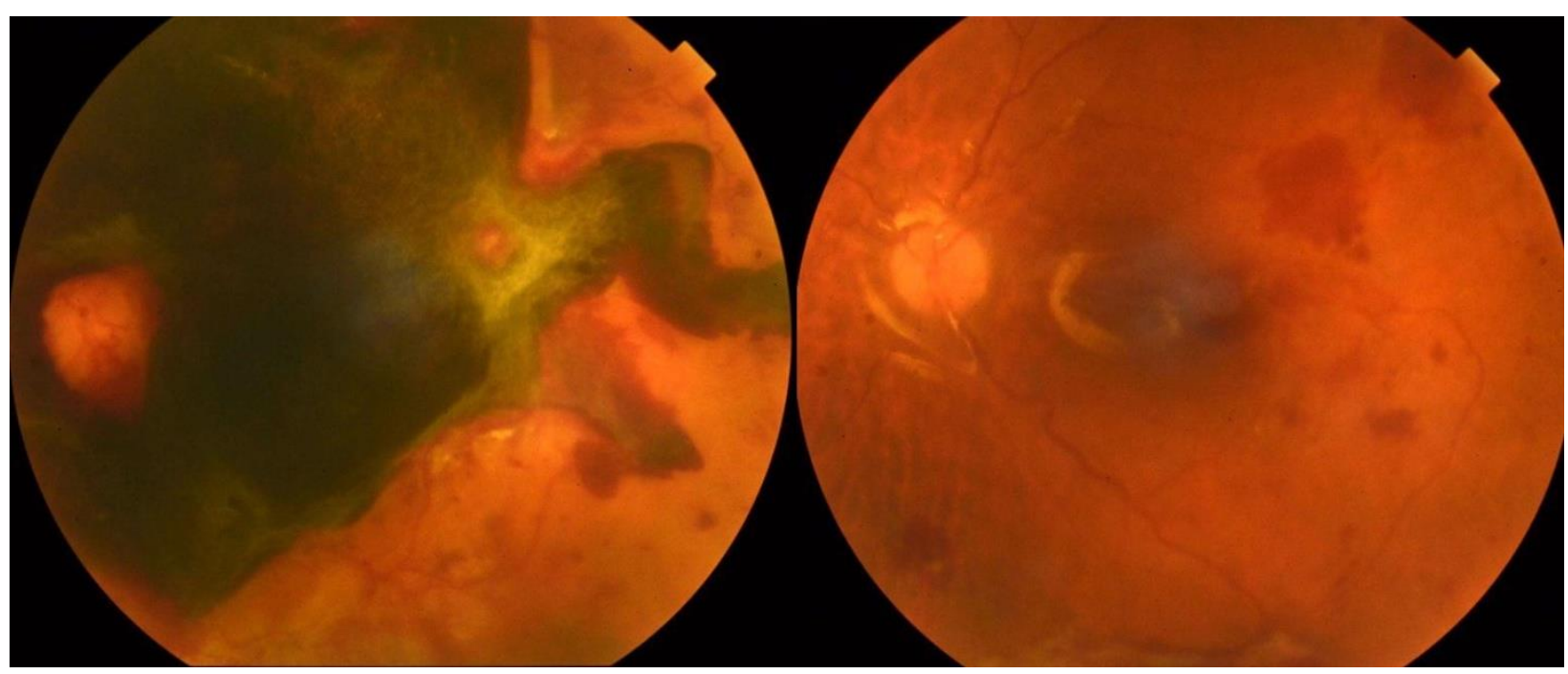

Figure 2: Fundus picture of theleft eye of 55 years old male with premacular hemorrhage (Sub-epiretinal membrane) due to proliferative diabetic retinopathy (right image) which is completely resolved after vitrectomy (left image). 


\section{DISCUSSION}

The main goal of treatment is to get rid off the hemorrhage. The majority of patients who presented with vitreous and pre-retinal hemorrhages do not need surgical intervention because of spontaneous resolution. Although we can observe it for about four weeks or even more, especially in sub-ILM hemorrhage, to get spontaneous absorption of premacular hemorrhage, some cases fail to resolve and hence other treatment modalities should be tried ${ }^{(12)}$.Pulsed Nd-YAG membranotomy (hyaloidotomy) was tried before and it provides another good alternative for patients presenting with subhyaloid hemorrhage. However it carries the risk of retinal detachment and macular hole formation, and formation of epimacular gliotic membranes as a wound-healing reaction especially in eyes with sub-ILM hemorrhage as a complications. Furthermore, this technique cannot be practiced in young children under five years and in babies as well as in eyes with dense macular hemorrhage ${ }^{(1,2,4,8,11,13-15)}$.

Intravitreal tissue plasminogen activator with sulfur hexafluoride injection to induce pneumatic displacement of the premacular blood away from the macula with subsequent its absorption, is another way for treatment of premacular hemorrhage, but it may need multiple injections and carry some complications ${ }^{(16-18)}$.

Longstanding cases of premacular hemorrhage may be complicated by the formation of epimacular membranes and the development of proliferative vitreoretinopathy especially in diabetic patients. These cases need pars plana vitrectomy as it will remove the gliotic tissue in front of the macula with rapid regain of macular function. Macular traction may also develop after spontaneous resorption of the premacular hemorrhage, which again will need vitrectomy ${ }^{(19)}$.

Pars plan vitrectomy, when done by a qualified surgeon, is another option for treatment of premacular hemorrhage and serves a safe and effective alternative to observation and other lines of treatment as it facilitates rapid visual recoveryafter one surgery ${ }^{(10,20)}$.

In this study pars plana vitrectomy was done in 17 patients presented with premacular hemorrhage of different pathologies. Clearing up of the blood was achieved in all patients. During vitrectomy nine cases out of seventeen were subhyaloid hemorrhage while five cases were sub-ILM hemorrhage and three cases were sub-epiretinal membrane (ERM).
ILM peeling was done in nine cases including all cases of sub-ILM hemorrhage. All patients achieved an improvement of their visual acuity from hand motion before surgery to an average of $0.4 \pm 0.22$ within one-month postoperatively. All cases of Valsalva gained the higher visual improvement whereas the cases of diabetic retinopathy had the lowest visual improvement due to diabetic maculopathy.

In 2007, pars plana vitrectomy was performed by some authors in five patients presented with premacular hemorrhage. Intraoperatively, they found that sub-ILM hemorrhage was present in all patients. Also, they recorded an improvement of visual functions in the five patients without complications related to pars plana vitrectomy ${ }^{(21)}$.

O'Hanley etal., also reported an improvement of visual acuity in all patients presented with dense premacular hemorrhage secondary to proliferative diabetic retinopathy after vitrectomy when performed within 4 weeks of the onset of the hemorrhage. While they observed that patients who did not have vitrectomy within 4 weeks of the onset of the hemorrhage developed macular traction with an impairment of their final visual acuity ${ }^{(22)}$.

In our study,intraoperative complications were recorded in table (3). Postoperative posterior subcapsular cataract was developed in four $(44.5 \%)$ of nine phakic patients as shown in table (4), for two of them phacoemulsification with PC IOL was done four months post-vitrectomy and the other two cases did not need cataract surgery during the follow up time. Recurrent vitreous hemorrhage happened in two cases two months postoperativelyand was related to the original pathology (diabetic retinopathy) and treated medically. No retinal detachment was reported in our study. Our incidence of complications is consistent with the reported vitrectomy complications in the literature ${ }^{(23-27)}$.

Although surgical evacuation of the premacular hemorrhage at the proper time has the advantage of immediate visual recovery and rehabilitation. However, vitrectomy, despite being a safe procedure especially with the advent of small gauge sutureless vitrectomies, still carries the risk of some complications $^{(27)}$.

\section{CONCLUSION}

In summary, the age of the patient, underlying pathology, density, size, and duration of the hemorrhage are some of the factors that affect the 
decision making in the treatment of premacular hemorrhage.Either waiting for spontaneous resorption or doing vitrectomy will be affected by these factors.

Further studies with a large number of patients will be needed to confirm the effectiveness and the safety of vitrectomy in patients presenting with premacular hemorrhage.

\section{REFERENCES}

1. Kaynak S, Eryildirim A, Kaynak $T$ et al.(1994): $\mathrm{Nd}: \mathrm{YAG}$ laser posterior hyaloidotomy in subhyaloid hemorrhage. Ophthalmic Surg, 25: 474-6.

2.Adel B, Friedman $Z$ (1998): Dense subhyaloid hemorrhage or subinternal limiting membrane hemorrhage in the macula treated by Nd:YAG laser. Arch Ophthalmol. , 116: 1542-3.

3. Khairallah M,Ladjimi A,Messaoud R,Yahia SB,Hmidi K,Jenzeri S(1999): Retinal venous macroaneurysm associated with premacular hemorrhage. Ophthalmic SurgLasers , 30: 226-8.

4. Ulbig MW, Mangouritsas G, Rothbacher HH, Hamilton AM, McHugh JD(1998):Long-term results after drainage of premacular subhyaloid hemorrhage into the vitreous with a pulsed Nd:YAGlaser.ArchOphthalmo.1,116:1465-9.

5. Spraul CW, Grossniklaus HE(1997): Vitreous hemorrhage.Surv Ophthalmol. ,42:3-39.

6. Schmidt JC, NietgenGW(1998): Argon laser treatment of subhyaloidal hemorrhage. Laser medizin, 14:94-8.

7. Shukla D, Naresh KB, Kim R(2005): Optical coherence tomography findings in valsalva retinopathy. Am $\mathrm{J}$ Ophthalmol.,140:134-6.

8. Raymond LA(1995): Neodymium:YAG laser treatment for hemorrhages under the internal limiting membrane and posterior hyaloid face in the macula. Ophthalmology, 102: 406-11.

9. Messmer EP, Wessing A, Ruprecht K, umann GO(1984): Solitary intraretinal macular hemorrhage. Graefe's Arch Clin Exp Ophthalmol., 222:9-12.

10. Kuhn F, Morris R, Witherspoon CD, Mester V(1998):Terson syndrome. Results of vitrectomy and the significance of vitreous hemorrhage in patients with subarachnoid hemorrhage.Ophthalmology, 105:472-7.

11. Gabel VP, Birngruber R, Gunther-Koszka H,Puliafito CA(1989): Nd:YAG laser photodisruption of hemorrhagic detachment of the internal limiting membrane. Am J Ophthalmol.,107:566-8.

12. Morris R, Kuhn F, Witherspoon CD, Mester V,Dooner J(1997):Hemorrhagic macular cysts in Terson's syndrome and its implications for macular surgery. In: Wiedemann P, Kohen L (eds) Macular and retinal diseases. Dev Ophthalmol series, 29: 44-54.
13. Kwok AK, Lai TY, Chan NR(2003): Epiretinal membrane formation with internal limiting membrane wrinkling after Nd: YAG laser membranotomy in valsalva retinopathy. Am J Ophthalmol.,136:763-6

14. Thach AB, Lopez PF, Snady-McCoy LC, Golub BM, Frambach D(1995): Accidental Nd:YAG laser injuries to the macula.Am J Ophthalmol., 119: 763-73.

15. Alhalel A, Glovinsky Y, Treister G, Bartov E, Blumenthal M, Belkin M(1993): Long-term follow-up of accidentalparafoveal laser burns. Retina, 13: 152-4.

16. Chou YK, Huang YM, Lin PK(2015): Sub-internal limiting membrane hemorrhage treated with intravitreal tissue plasminogen activator followed by octafluoropropanegasinjection.Taiwan Journal of Ophthalmology ,5: 198-201

17. Mizutani T, Yasukawa T, Ito $Y$ et al.(2011): Pneumatic displacement of submacular hemorrhage with or without tissue plasminogen activator. Graefes Arch Clin Exp Ophthalmol.,249:1153-7.

18. Singh RP, Patel C, Sears JE(2006): Management of subretinal macular haemorrhage by direct administration of tissue plasminogen activator. $\mathrm{Br} \mathrm{J}$ Ophthalmol. ,90:429-431.

19. Gibran SK, Kenawy N, Wong D, Hiscott $\mathbf{P}(2007)$ : Changes in the retinal inner limiting membrane associated with Valsalva retinopathy. Br JOphthalmol.,91:701-2.

20. Meier P, Schmitz F, Wiedemann P(2005): Vitrectomy for pre-macular hemorrhagic cyst in children and young adults. Graefe's Arch Clin Exp Ophthalmol.,243:824-8.

21. De Maeyer K, Van Ginderdeuren R, Postelmans L, Stalmans P, Van Calster J(2007):Subinner limiting membrane haemorrhage: causes and treatment with vitrectomy.Br J Ophthalmol. ,91:869e872.

22. O'Hanley GP, Canny CL(1985): Diabetic dense premacular hemorrhage. A possible indication for prompt vitrectomy. Ophthalmology ,92:507e511.

23. Kim KY, Yu SY, Kim M, Kwak HW(2014): Macular hole formation after parsplana vitrectomy for the treatment of Valsalva retinopathy: a case report.Korean J Ophthalmol.,28:91-95.

24. Carter JB, Michels RG, Glaser BM, de Bustros S(1990): Iatrogenic retinal breaks complicating pars plana vitrectomy. Ophthalmology ,97:848-53.

25. Ghoraba HH, Zayed AI(2001):Suprachoroidal hemorrhage as a complication of vitrectomy. Ophthalmic Surg.Lasers, 32:281- 8 .

26. Eifrig CW, Scott IU, Flynn HW,Smiddy WE, Newton J(2004):Endophthalmitis after pars plana vitrectomy: incidence, causative organisms, and visual acuity outcomes. Am.J.Ophthalmol.,138:799-802.

27. Jackson TL, Thiagarajan M, Murthy R, Snead MP, Wong D, Williamson TH(2001): Pupil block glaucoma in phakic and pseudophakic patients after vitrectomy with silicone oil injection. Am.J.Ophthalmol., 132:414-6. 